\title{
Inflammasome-mediated neurodegeneration following heart disease
}

\author{
Kuan Cheng ${ }^{1 \#}$, Jingjing Wang ${ }^{2 \#}$, Qingxing Chen ${ }^{1}$, Gang Zhao ${ }^{1}$, Yang Pang ${ }^{1}, \mathrm{Ye} \mathrm{Xu}^{1}$, Junbo Ge ${ }^{1}$, \\ Wenqing $\mathrm{Zhu}^{1}$
}

${ }^{1}$ Department of Cardiology, Zhongshan Hospital, Fudan University, Shanghai Institute of Cardiovascular Diseases, Shanghai, China; ${ }^{2}$ Department of Emergency, Shanghai Pulmonary Hospital, Tongji University, Shanghai, China

Contributions: (I) Conception and design: K Cheng; (II) Administrative support: None; (III) Provision of study materials or patients: K Cheng, W Zhu; (IV) Collection and assembly of data: All authors; (V) Data analysis and interpretation: K Cheng, W Zhu; (VI) Manuscript writing: All authors; (VII) Final approval of manuscript: All authors.

\#These authors contributed equally to this work.

Correspondence to: Wenqing Zhu. Department of Cardiology, Zhongshan Hospital, Fudan University, Shanghai Institute of Cardiovascular Diseases, 1609 Xietu Road, Shanghai 200032, China. Email: zwq4563@yeah.net.

Background: Myocardial infarction (MI) has been suggested as a critical predisposing factor for Alzheimer's disease (AD); however, the underlying mechanisms remain unknown. PYD domains-containing protein 3 (NLRP3) is a key factor to mediate inflammasome formation. Previous studies have shown that NLRP3 activation in brain microglia is required for AD; however, its possible role in MI-induced future development of neurodegeneration is not yet understood. These questions were addressed in the current study.

Methods: We generated microglia-specific NLRP3 mutation mice in the AD-prone APP/PS1 background (APP/PS1/NLRP3 ${ }^{\mathrm{MKO}}$ ), and studied the neurodegeneration in these mice after MI compared to the control wild-type C57/BL6J or APP/PS1 mice. NLRP3, IL-1 $\beta$ and caspase-1 levels were determined by Enzymelinked immunoassay (ELISA). The heart function was determined by the slope of end systolic pressurevolume relationship, left ventricular end diastolic pressure, the positive maximal pressure derivative as well as the degree of fibrosis by Masson's trichrome staining. Mouse behavior measurement includes Morris watermaze test and motor assessment.

Results: We found that compared with the control wild-type C57/BL6J or APP/PS1 mice, the effects of $\mathrm{MI}$ on the subsequent development of defected spatial reference memory and motor activity were all attenuated in APP/PS1/NLRP3 ${ }^{\mathrm{MKO}}$ mice, likely resulting from the reduced formation of amyloid-beta peptide aggregates $(\mathrm{A} \beta)$ plaques.

Conclusions: NLRP3 may play a non-redundant role in the MI-induced future development of AD.

Keywords: Inflammasome; PYD domains-containing protein 3 (NLRP3); Alzheimer's disease (AD); myocardial infarction (MI)

Submitted Aug 22, 2021. Accepted for publication Oct 16, 2021.

doi: $10.21037 / \mathrm{atm}-21-4931$

View this article at: https://dx.doi.org/10.21037/atm-21-4931

\section{Introduction}

Alzheimer's disease (AD) is characterized by accelerated deposit of extracellular amyloid-beta peptide aggregates $(A \beta)$ plaques and augmented formation of intraneuronal neurofibrillary tangles (NFTs) by hyperphosphorylated
Tau (pTau) proteins. As a prevalent disease in aged people, $\mathrm{AD}$ patients typically exhibit behavioral disorders such as compromised memory, learning potential, and locomotive capability (1).

Recently, myocardial infarction (MI) has been suggested 
as a critical predisposing factor for $\mathrm{AD}$ (2). Specifically, $\mathrm{AD}$ and MI share a similar genetic background, implying a link between these two diseases (3). Moreover, a cohort study has shown an association between higher risk of vascular dementia and MI (4). However, the underlying mechanisms remain poorly defined.

A proinflammatory cytokine, interleukin $1 \beta$ (IL-1 $\beta$ ), plays a profound role in a variety of diseases, including $\mathrm{AD}(5-7)$. The elucidated mechanisms by which biologically active IL$1 \beta$ is made in the cell usually involve activation of a protein complex called the inflammasome (8). Inflammasomes are typically formed by a member of the Nod-like receptor (NLR) family that oligomerizes the apoptosis-associated speck-like protein containing a caspase-recruitment domain (ASC) to dimerize and activate caspase-1, which subsequently cleaves the precursor form of IL- $1 \beta$ (pro-IL-1 $\beta$ ) into the processed, secreted form, IL-1 $\beta$ (9). PYD domainscontaining protein 3 (NLRP3) is the most important enzyme that activates NLR family members for the formation and assembly of the inflammasome complex (10). Interestingly, previous studies have shown that NLRP3 activation in brain microglia is required for the progression of neurodegenerative diseases, including $\mathrm{AD}$ (11). Moreover, inhibition of the NLRP3 inflammasome with MCC950 promotes non-phlogistic clearance of amyloid- $\beta$ and cognitive function in APP/PS1 mice (12). However, none of these studies have addressed a specific role of NLRP3 in microglia. In addition, whether NLRP3-mediated activation of an inflammasome in microglia plays a role in the MI-induced future development of neurodegeneration is not currently known. We present the following article in accordance with the ARRIVE reporting checklist (available at https://dx.doi.org/10.21037/atm-21-4931).

\section{Methods}

\section{Experimental and animal protocols}

Experiments were performed under a project license (approved at 03/27/2021, same title with this article) granted by institutional ethics board of Fudan University. All experiments were performed in strict accordance with Guidelines for Laboratory Animal Management issued by Fudan University. A protocol was prepared before the study without registration. Wild-type (WT) C57/BL6J mice, APPswe/PSEN1dE9 (APP/PS1; \#025970) (13), Tmem1192A-CreERT2 (\#031820) (14), and conditional NLRP3 mutation (\#017969) (15) transgenic mice were all purchased from Jackson Labs (Bar Harbor, ME, USA). APP/PS1 mice are double transgenic mice expressing a chimeric mouse/ human amyloid precursor protein (Mo/HuAPP695swe) and a mutant human presenilin 1 (PS1-dE9), both directed to CNS neurons to induce early-onset Alzheimer's disease. Tmem119-2A-CreERT2 and conditional NLRP3 mutation mice were first bred to generate microglia-specific NLRP3 knockout mice NLRP3 ${ }^{\mathrm{MKO}}$, and subsequently bred to APP/ PS1 mice to generate APP/PS1/NLRP $3^{\mathrm{MKO}}$ mice. WT and APP/PS1 mice were used as controls.

The mice were individually housed under a 12 -hour light-dark cycle (temperature: $24 \pm 2{ }^{\circ} \mathrm{C}$; humidity: $46 \% \pm 4 \%$ ) with ad libitum access to water and food. Female and male mice were randomly and evenly distributed in each experimental group, and did not exhibit intra-group differences in the experimental tests. Mice at 2.5 months of age (female around 23-26 g and male around 31-35 g) were subjected to $\mathrm{MI} / \mathrm{sham}$ surgery and followed-up for another 2.5 months (until the age of 5 months old) for analysis of $\mathrm{AD}$ development.

For each experiment, Power calculations $(\mathrm{P}<0.05)$ were performed in order to include sufficient numbers of animals, in order to be confident that the observed effects were real. An allocation concealment method was used to provide randomization in allocating experimental units to the control and treatment groups. Since inbred littermate mice were used in a specified experiment, the potential confounders were minimized. No criteria were used for excluding animals (or experimental units) during the experiment, and no data were excluded during the analysis. The study did not have humane endpoints.

\section{MI in mice and analysis of heart function}

MI was induced in mice at 2.5 months of age by ligation of the left anterior descending artery with 9-0 nylon sutures in the middle, after anesthetization with isoflurane (dose: $4.5 \%$ for induction; $2 \%$ for maintenance). Coronary occlusion was confirmed by the blanching of the apex. The shamoperated group received only left thoracotomy. The chest was closed with 5-0 sutures. To determine heart function, a volume catheter of $1.2 \mathrm{~F}$ pressure was connected to an Advantage PV-loop system (Transonic Systems Inc., Ithaca, NY, USA), and then inserted into the left ventricle via the right carotid artery. Hemodynamic assessment, including end systolic pressure-volume relationship (ESPVR slope), left ventricular end diastolic pressure (LVESP), and positive maximal pressure derivative $(+\mathrm{dP} / \mathrm{dt})$ were used to validate 
model establishment.

\section{Behavioral analysis}

Spatial reference memory was measured using the Morris water-maze test starting 2.5 months after sham/MI surgery or at 5 months of age. Briefly, a circular pool $(50 \mathrm{~cm}$ in diameter) was filled with opaque water to a depth of $30 \mathrm{~cm}$. A platform $(5 \mathrm{~cm}$ in diameter) was submerged $2 \mathrm{~cm}$ below the surface in the center of the target quadrant. Surrounding permanent visual cues were used for spatial orientation. The time needed by the mice to reach the hidden platform was recorded on days $1,3,5,7$, and 9 . The swim speed of the mice was also recorded. Moreover, the complete platform was divided into four quadrants, while the real platform was located in quadrant 1 . The routine by which the mice ran was recorded and the percentage of the route that the mice spent in quadrant 1 was calculated.

Motor assessment was conducted in parallel. First, all mice were tested using a grip test to measure the maximal strength with which a mouse was able to hold a $300 \mathrm{~g}$ bar with its front paws while an experimenter pulled it gently backwards. Deficits in limb coordination and accuracy of limb placement, as well as general motor coordination of the mice, was evaluated using the beam crossing test, which involves a mouse being guided to walk across a beam to reach a home cage. Briefly, the beam apparatus consisted of a 1-meter beam with a flat surface $(1.5 \mathrm{~cm}$ wide). A black box with nesting material was placed at the end of the beam as the finish point to attract the mouse. A lamp (with 60 watt light bulb) was used to shine light above the start point and served as an aversive stimulus. The time to cross the beam was recorded.

\section{Histology, immunobistochemistry, and ELISA}

Following heart perfusion with $4 \%$ formalin, the mouse hearts and brains were fixed in $4 \%$ formalin for 4 and 6 hours, respectively, and then embedded in paraffin. Serial sections were cut at 5 microns, mounted, and rehydrated according to standard protocols. Hematoxylin counterstaining was used to provide cytologic detail. The infarcted heart tissue was stained using Masson's trichrome method (HT-15-1KT, Sigma-Aldrich, Beijing, China). The cornu ammonis 1 (CA1) region of the mouse hippocampus was specially embedded and cut for analysis. The mouse hippocampus was located in the temporal lobe below the cerebral cortex. CA1 is the first region in the hippocampal circuit, from which a major output pathway goes to layer $\mathrm{V}$ of the entorhinal cortex. In the current study, we focused on examining the CA1 region of the mouse brain.

Immunostaining for determining neuronal cell number was performed using a rabbit anti-NeuN antibody (ab177487, Abcam, Dallas, Texas, USA). To measure neuronal cell loss, NeuN staining was performed and the density of NeuN + cells was calculated $\left(\right.$ cells $/ \mathrm{mm}^{3}$ ) in a systematic random fashion. To determine $A \beta$ deposition, the brain tissue was stained with a specific $A \beta$ antibody (ab201060, Abcam) using DAB (3,3'-diaminobenzidine) staining method, and the $A \beta+$ area was counted. Enzymelinked immunoassay (ELISA) for NLRP3 (LS-F32087, LSBio, Seattle, WA, USA), IL-1 $\beta$ (DY401, R\&D System, Los Angeles, CA, USA), and caspase-1 (LS-F33112, LSBio) were carried out using specific kits as instructed.

\section{Statistical analysis}

Statistical analysis was performed using GraphPad Prism 8.2 (GraphPad Software, San Diego, CA, USA). Analysis of variance (ANOVA) was conducted by one-way ANOVA with a Bonferroni correction, followed by Fisher's exact test upon necessity. All values are depicted as mean \pm standard deviation (SD) and are considered significant if $\mathrm{P}<0.05$. Non-significance is presented as NS.

\section{Results}

\section{MI activates brain inflammasomes in APP/PS1 mice but not in APP/PS1/NLRP $3^{\text {MKO }}$ mice}

Tmem119-2A-CreERT2 and conditional NLRP3 mutation mice were first bred to generate microglia-specific NLRP3 knockout mice NLRP3 ${ }^{\mathrm{MKO}}$, and then bred to APP/PS1 mice to generate APP/PS1/NLRP3 ${ }^{\mathrm{MKO}}$ mice. WT C57/BL6J and APP/PS1 mice were used as controls. The reason that we generated microglia-specific NLRP3 mutant mice is that NLRP3 has been reported to be essential for pathogenetic progression in MI and subsequent reperfusion (16). The groups were then set up as follows: Group 1: WT mice that were sham-operated; Group 2: WT mice that received MI operation; Group 3: APP/PS1 mice that were sham-operated; Group 4: APP/PS1 mice that received MI operation; Group 5: APP/PS1/NLRP3 ${ }^{\mathrm{MKO}}$ mice that were sham-operated; and Group 6: APP/PS1/NLRP3 ${ }^{\mathrm{MKO}}$ mice that received MI operation. All of the mice were operated on at 2.5 months of age. 

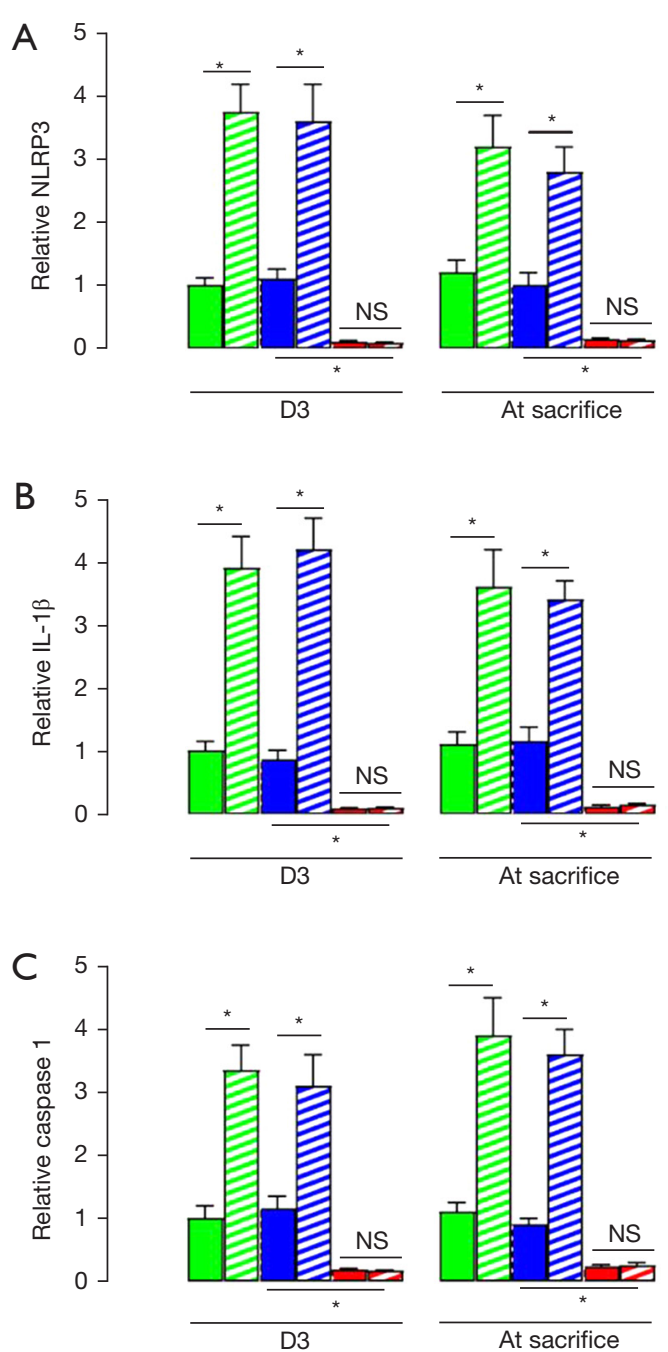
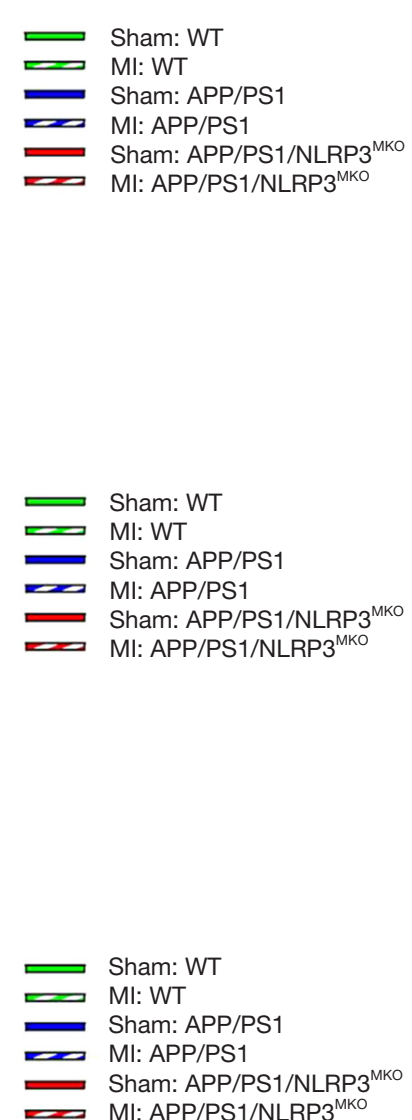

Figure $1 \mathrm{MI}$ activates brain inflammasomes in APP/PS1 mice but not in APP/PS1/NLRP3 ${ }^{\text {MKO }}$ mice. Tmem119-2A-CreERT2 and conditional NLRP3 mutation mice were first bred to generate microglia-specific NLRP3 knockout mice NLRP $3^{\mathrm{MKO}}$, and then bred to APP/ PS1 mice to generate APP/PS1/NLRP3 ${ }^{\mathrm{MKO}}$ mice. Wild-type (WT) C56/BL6J and APP/PS1 mice were used as controls. The groups were then set up as follows: Group 1: WT mice that were sham-operated; Group 2: WT mice that received MI operation; Group 3: APP/PS1 mice that were sham-operated; Group 4: APP/PS1 mice that received MI operation; Group 5: APP/PS1/NLRP3 ${ }^{\mathrm{MKO}}$ mice that were shamoperated; and Group 6: APP/PS1/NLRP $3^{\text {MKO }}$ mice that received MI operation. All of the mice were operated on at 2.5 months of age. Mice were sacrificed and their brains were assessed for expression of NLRP3 (A), IL-1 $\beta$ (B), and caspase-1 (C) by ELISA at 3 days after the MI/ sham operation, or at approximately 3 months after all of the experiments were performed when the mice were sacrificed. *, $\mathrm{P}<0.05$. NS, non-significant. Ad day 3, $\mathrm{N}=4$; at sacrifice, $\mathrm{N}=9$.

Three days after the MI/sham operation, some mice were sacrificed and their brain was assessed for expression of NLRP3, IL-1 $\beta$, and caspase-1. First, microglia from 3 experimental mouse strains were isolated based on their expression of Tmem119 and examined for NLRP3 levels by ELISA, which confirmed the knockdown of NLRP3 specifically in APP/PS1/NLRP $3{ }^{\mathrm{MKO}}$ mice (Figure S1). We detected significant increases in brain NLRP3 in both WT and APP/PS1 mice after MI, compared to the corresponding sham-operated mice (Figure 1A). Brain NLRP3 levels were extremely low in APP/PS1/NLRP3 ${ }^{\text {MKO }}$ mice and did not increase after MI (Figure 1A). Similarly, we 


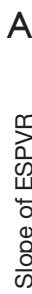

व

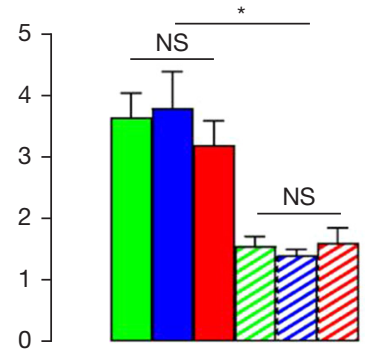

B
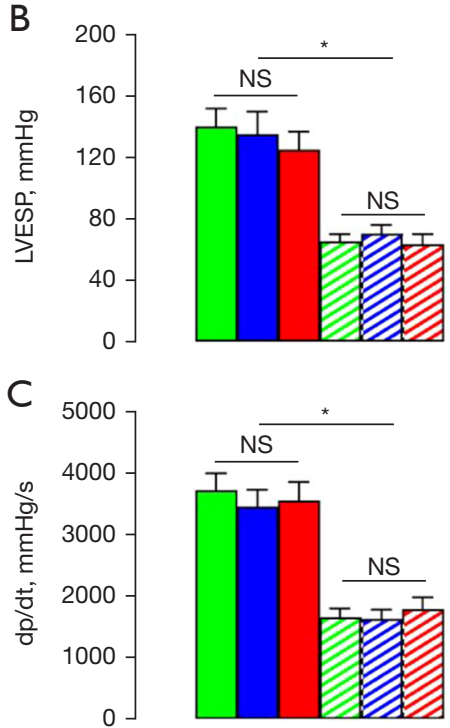

$$
\begin{aligned}
& \text { Sham: WT } \\
& \text { Sham: APP/PS1 } \\
& \text { Sham: APP/PS1/NLRP3 }{ }^{\text {MKo }} \\
& \text { Ml: WT } \\
& \text { Ml: APP/PS1 } \\
& \text { Ml: APP/PS1/NLRP3 }
\end{aligned}
$$

D

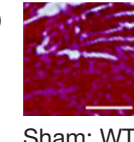

Sham: WT

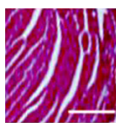

Sham: APP/PS1

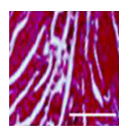

Sham: APP/PS1/NLRP3 ${ }^{\text {MKo }}$

$$
\begin{aligned}
& \text { Sham: WT } \\
& \text { Sham: APP/PS1 } \\
& \text { Sham: APP/PS1/NLRP3 }{ }^{\text {Mко }} \\
& \text { Ml: WT } \\
& \text { Ml: APP/PS1 } \\
& \text { Ml: APP/PS1/NLRP3 }{ }^{\text {Mко }}
\end{aligned}
$$

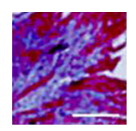

MI: WT

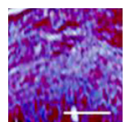

MI: APP/PS1

$$
\begin{aligned}
& \text { Sham: WT } \\
& \text { Sham: APP/PS1 } \\
& \text { Sham: APP/PS1/NLRP3 }{ }^{\text {мко }} \\
& \text { Ml: WT } \\
& \text { Ml: APP/PS1 } \\
& \text { Ml: APP/PS1/NLRP3 } \\
&
\end{aligned}
$$

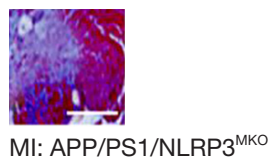

Figure $2 \mathrm{MI}$ causes heart dysfunction in all experimental mice. The behavior of the mice was analyzed over a 2 -week period at 2.5 months after MI, and the mice were then subjected to heart function assessment. Assessment of (A) the slope of ESPVR, (B) LVESP, and (C) positive maximal pressure derivative $(+\mathrm{dP} / \mathrm{dt}$ ). (D) Representative Masson's trichrome staining of the mouse heart 2.5 months after sham/ $\mathrm{MI}$ at sacrifice. We found that a similar degree of MI was induced in APP/PS1/NLRP3 ${ }^{\mathrm{MKO}}$, APP/PS1, and WT mice. *, P<0.05. NS, nonsignificant. For groups 1 and 2 (WT), N=5. For groups 3-6 (APP/PS1/NLRP3 ${ }^{\text {MKO }}$, APP/PS1), N=9. Scale bars are $50 \mu$.

detected marked increases in brain IL-1 $\beta$ in both $\mathrm{WT}$ and APP/PS1 mice after MI, compared to the corresponding sham-operated mice (Figure 1B). Brain IL-1 $\beta$ levels were extremely low in APP/PS1/NLRP $3^{\mathrm{MKO}}$ mice and did not increase after MI (Figure 1B). Also, we detected notable increases in brain caspase-1 in both WT and APP/PS1 mice after $\mathrm{MI}$, compared to the corresponding sham-operated mice (Figure 1C). Brain caspase-1 levels were extremely low in APP/PS1/NLRP ${ }^{\text {MKO }}$ mice and did not increase after MI (Figure 1C). Interestingly, the expression pattern was maintained for approximately 3 months after the experiments when the mice were sacrificed (Figure 1A-1C). These results are consistent with a regulatory axis of NLRP3/caspase-1/IL-1 $\beta$, and confirm a microglia-specific loss of NLRP3 in APP/PS1/NLRP $3^{\text {MKO }}$ mice.

\section{MI causes beart dysfunction in all experimental mice}

The behavior of the mice was analyzed over a 2 -week period at 2.5 months after MI, and the mice were subsequently subjected to heart function assessment. We observed that $M I$ induced significant and similar decreases in the slope of ESPVR (Figure 2A), LVESP (Figure 2B), and positive maximal pressure derivative $(+\mathrm{dP} / \mathrm{dt})($ Figure $2 C)$ in all $\mathrm{WT}$, APP/PS1, and APP/PS1/NLRP3 ${ }^{\mathrm{MKO}}$ mice. The MI-induced pathological changes in the mouse heart were confirmed by Masson's trichrome staining at sacrifice $(2.5$ months + 2 weeks after sham/MI; Figure 2D). These results not only confirmed microglia-specific loss of NLRP3 in APP/PS1/ NLRP $3{ }^{\mathrm{MKO}}$ mice, since global loss of NLRP3 has been shown to attenuate MI-induced cardiac dysfunction (17), 
but also suggested that a similar degree of MI was induced in APP/PS1/NLRP3 ${ }^{\mathrm{MKO}}$, APP/PS1, and WT mice.

\section{APP/PS1/NLRP $3^{M K O}$ does not exhibit MI-induced aggravated spatial reference memory in APP/PS1 mice}

At 2.5 months after sham/MI, the sham- or MI-treated WT, APP/PS1/NLRP $3^{\text {MKO }}$, and APP/PS1 mice underwent behavioral tests over a 2 -week period. The WT did not exhibit impairment in these tests (Figure 3). In the Morris water-maze test for assessing spatial reference memory, MI significantly impaired the time needed by the APP/ PS1 mice to reach the platform (Figure $3 A$ ). The time for the mice to reach platform was reduced in APP/PS1/ $\mathrm{NLRP}^{\mathrm{MKO}}$ mice and did not increase after MI (Figure $3 A$ ). On the other hand, the swim speed of the mice was not different between APP/PS1/NLRP $3^{\mathrm{MKO}}$ and APP/PS1 mice, regardless of $M I$ treatment (Figure 3B).

Moreover, MI significantly reduced the route trace for the APP/PS1 mice to spend in the quadrant where the platform located (Figure 3C,3D). However, the route trace for the APP/PS1/NLRP3 ${ }^{\mathrm{MKO}}$ mice to spend in the quadrant where the platform located was significantly higher and was not reduced by MI (Figure 3C,3D). Together, these results suggest that spatial reference memory in APP/PS1/ NLRP3MKO mice was markedly better than that in APP/ PS1 mice, while MI impaired the spatial reference memory in APP/PS1 mice but not in APP/PS1/NLRP3MKO mice. In other words, MI-induced impairment of spatial reference memory was NLRP3-dependent.

\section{APP/PS1/NLRP $3^{\text {MKO }}$ does not exhibit MI-induced aggravated motor activity in APP/PS1 mice}

In the locomotor test for motor activity, the grip capability of the mice was not different between APP/ PS1/NLRP $3^{\mathrm{MKO}}$ and APP/PS1 mice, regardless of MI treatment (Figure 4A). MI significantly increased the time for the APP/PS1 mice to cross the beam (Figure 4B), while the time for the APP/PS1/NLRP3 ${ }^{\mathrm{MKO}}$ mice to cross the beam was significantly reduced and it did not increase after MI (Figure 4B). Thus, motor activity in APP/PS1/ NLRP3MKO mice was markedly better than that in APP/ PS1 mice, while MI impaired motor activity in APP/PS1 mice but not in APP/PS1/NLRP3MKO mice. In other words, MI-induced impairment of motor activity was NLRP3-dependent.

\section{MI-mediated AB deposition is NLRP3-dependent}

Finally, we studied the underlying mechanisms. We quantified neuronal loss in the hippocampus region of $\mathrm{WT}$, $\mathrm{APP} / \mathrm{PS} 1 / \mathrm{NLRP}^{\mathrm{MKO}}$, and APP/PS1 mice at sacrifice by NeuN staining. We found that MI did not significantly alter neuron density in WT mice (Figure $5 A, 5 B$ ). However, MI significantly reduced the neurons in APP/PS1 mice (Figure $5 A, 5 B$ ). The neurons in APP/PS1/NLRP $3^{\mathrm{MKO}}$ mice were significantly higher compared to APP/PS1 mice and did not decrease after MI (Figure 5A,5B). We also analyzed the $A \beta$ deposition in the mouse hippocampus of these mice at sacrifice, and found that MI did not increase $A \beta$ deposition in WT mice, but significantly increased $\mathrm{A} \beta$ levels in APP/PS1 mice (Figure 5C,5D). The A $\beta$ levels in APP/ PS1/NLRP $3^{\text {MKO }}$ mice were significantly lower than in APP/ PS1 mice and did not decrease after MI (Figure 5C,5D). Thus, NLRP3-dependent $A \beta$ deposition may be responsible for MI-induced neurodegeneration.

\section{Discussion}

Previous studies have shown that MI increases the likelihood of future development of $\mathrm{AD}$ in both mice and humans (18-20). On the other hand, NLRP3-mediated formation of an inflammasome has been shown to accelerate the progression of AD patients (11). Thioredoxin-interacting proteinassociated activation of the NLRP3 inflammasome has been detected in the brains of $\mathrm{AD}$ patients (21). Moreover, NLRP3 was revealed as a regulator of $A \beta$ autophagy in a mouse model of AD (22). Nevertheless, whether MI may promote development of AD via activation of the NLRP3 inflammasome remains an unanswered question.

To address this question, we generated microglia-specific NLRP3 mutation mice, since NLRP3 is activated in the heart during MI (16). Tmem119-2A-CreERT2 and conditional NLRP3 mutation mice were first bred to generate microgliaspecific NLRP3 knockout mice NLRP $3^{\mathrm{MKO}}$, and then bred to APP/PS1 mice to generate APP/PS1/NLRP $3^{\mathrm{MKO}}$ mice. WT and APP/PS1 mice were used as controls. Numerous studies have demonstrated that Tmem119 is microgliaspecific (14), and the Tmem119 promoter has been used to drive microglia-specific gene therapy (23).

In the present study, all of the mice received $M I$ at 2.5 months of age and were kept for another 2.5 months to be examined for development of $\mathrm{AD}$ using a set of behavior tests. After these tests, the mice were sacrificed for 
A
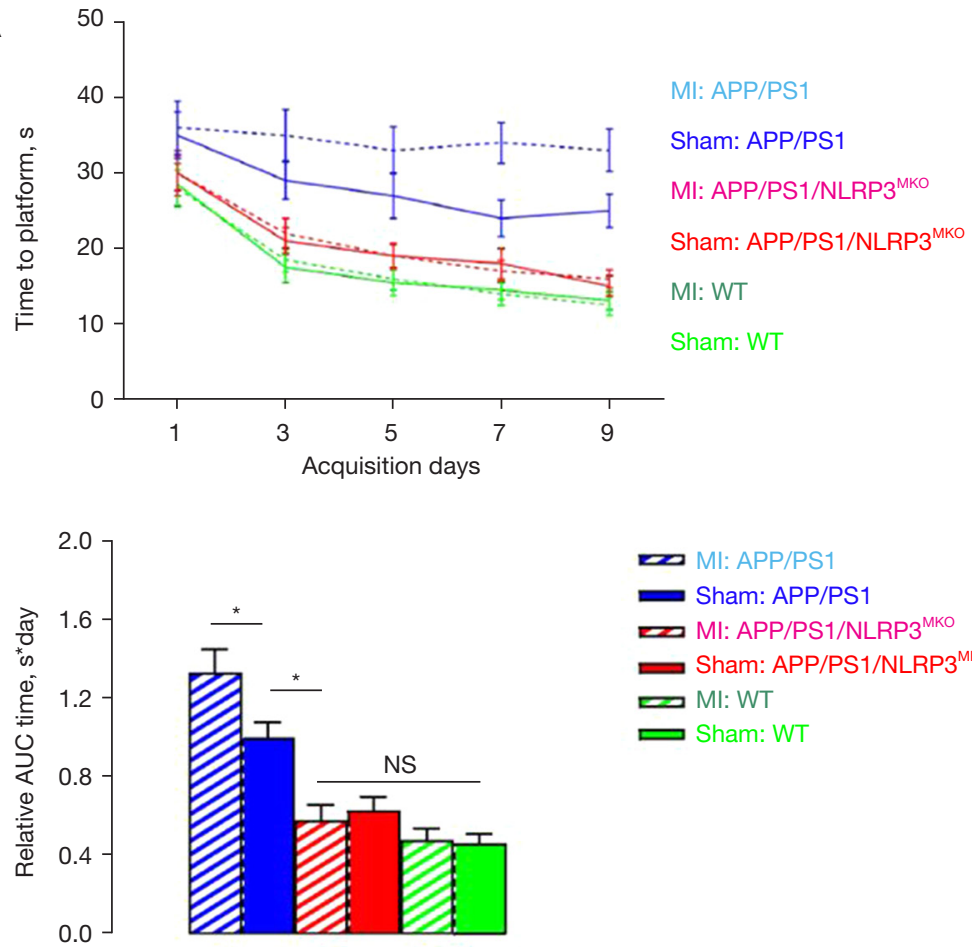

B

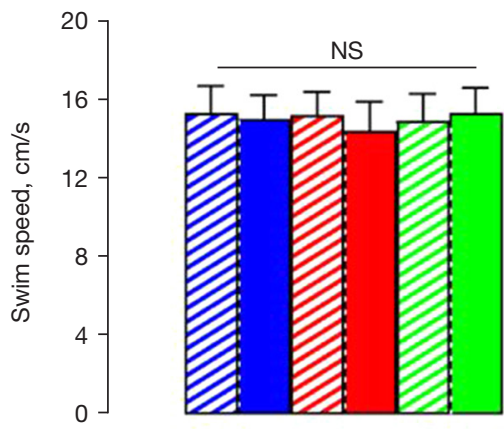

C

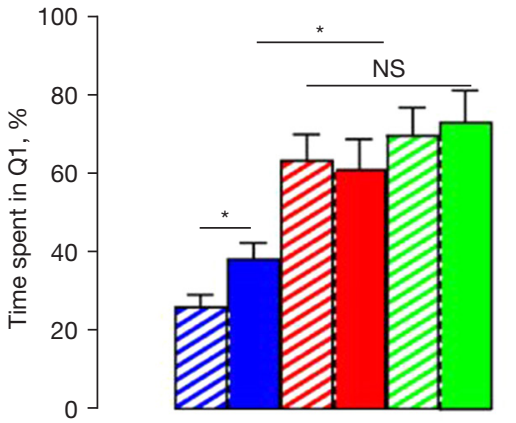

D
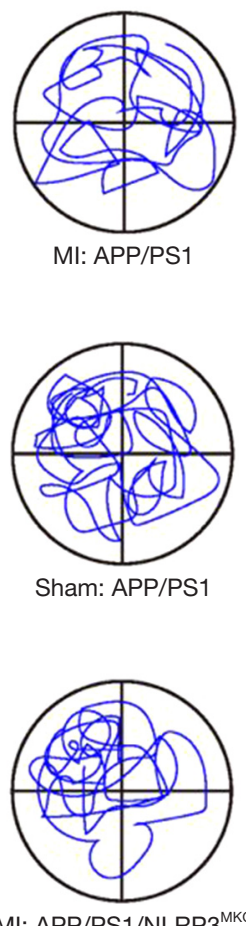

MI: APP/PS1/NLRP3 ${ }^{\text {MKO }}$
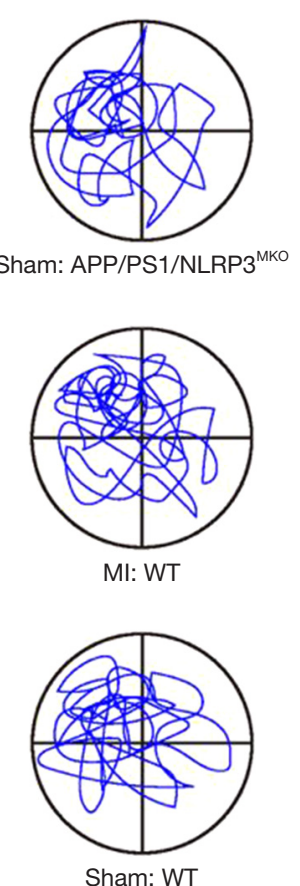

Figure 3 APP/PS1/NLRP3 ${ }^{\text {MKO }}$ does not exhibit MI-induced aggravated spatial reference memory in APP/PS1 mice. At 2.5 months after sham/MI, the sham- or MI-treated WT, APP/PS1/NLRP3 ${ }^{\text {MKO }}$, and APP/PS1 mice underwent a Morris water-maze test over a 2-week period. In a Morris water-maze test for assessing spatial reference memory, MI significantly impaired the spatial reference memory in APP/ PS1 mice, but MI did not affect it in WT or APP/PS1/NLRP3 ${ }^{\text {MKO }}$ mice. (A) The time for the mice to reach platform. (B) Swim speed of the mice. Route trace for the mice to spend in the quadrant where the platform located, by quantification (C) and by representative run curves (D). *, $\mathrm{P}<0.05$. NS, non-significant. For groups 1 and 2 (WT), N=5. For groups 3-6 (APP/PS1/NLRP3 $\left.{ }^{\mathrm{MKO}}, \mathrm{APP} / \mathrm{PS} 1\right), \mathrm{N}=9$. 
A

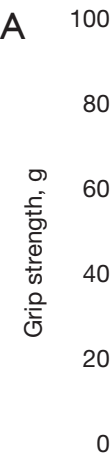

B

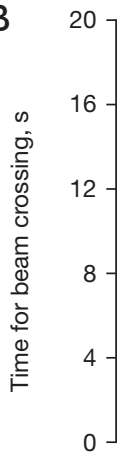

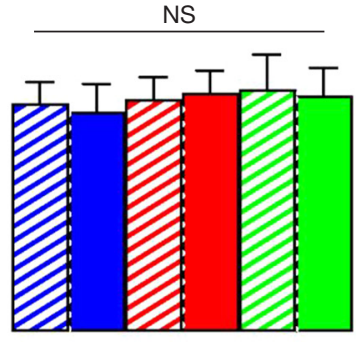

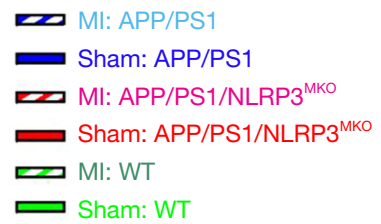

Figure 4 APP/PS1/NLRP3 ${ }^{\mathrm{MKO}}$ does not exhibit MI-induced aggravated motor activity in APP/PS1 mice. At 2.5 months after sham/MI, the sham- or MI-treated WT, APP/PS1/NLRP3 ${ }^{\mathrm{MKO}}$, and APP/PS1 mice underwent a locomotor test before sacrifice. MI significantly aggravated motor activity in APP/PS1 mice, but MI did not affect motor activity in WT or APP/PS1/NLRP3 ${ }^{\text {MKO }}$ mice. (A) The grip capability of the mice; (B) time to cross a beam. *, P<0.05. NS, non-significant. For groups 1 and 2 (WT), N=5. For groups 3-6 (APP/PS1/ NLRP $3^{\text {MKO }}$, APP/PS1), N=9.

histological assessment. This time course was determined based on the fact that the regular development of $\mathrm{AD}$ in APP/PS1 background is 5-6 months (13), as well as the typical requirement of more than 2 months for assessing the outcome of MI (16). We used both female and male mice in this study, but we did not observe significant differences in our analysis. Thus, we did not present data on male and female separately here.

The activation of NLRP3/caspase-1/IL-1 $\beta$ in the mouse brain immediately after MI suggested that the process of MI might directly affect inflammasome formation in the microglia in the brain, rather than affecting it indirectly and in the long term. The microglia-specific loss of NLRP3 in APP/PS1/NLRP3 ${ }^{\mathrm{MKO}}$ mice was also validated, as the MI-induced cardiac dysfunction did not differ between APP/PS1/NLRP $3^{\text {MKO }}$ mice and APP/PS1 mice. On the other hand, microglia-specific loss of NLRP3 in APP/ PS1/NLRP $3^{\mathrm{MKO}}$ mice appeared not to affect the severity of MI-induced cardiac dysfunction, which did not support presence of a feedback mechanism.

Microglia-specific loss of NLRP3 in APP/PS1/ $\mathrm{NLRP}^{\mathrm{MKO}}$ mice significantly prevented the development of spatial reference memory and motor activity defects, as well as MI-induced aggravation of defects in spatial reference memory and motor activity. This demonstrates that MI-induced development of $\mathrm{AD}$ requires the activation of NLRP3 in microglia, which likely induces inflammatory damage to neurons by facilitating $A \beta$ deposit.

Although our study provided strong evidence to indicate a requirement for NLRP3-dependent $A \beta$ deposition in microglia by $\mathrm{MI}$ for the future development of $\mathrm{AD}$ in mice, we did not experimentally prove that it could be same in human $\mathrm{AD}$ patients. Indeed, there is a significant difference in mouse $\mathrm{AD}$ models and human $\mathrm{AD}$ patients. $\mathrm{AD}$ is too complex to be properly and adequately modeled in mice. In fact, the "AD mice" only modeled some aspects 


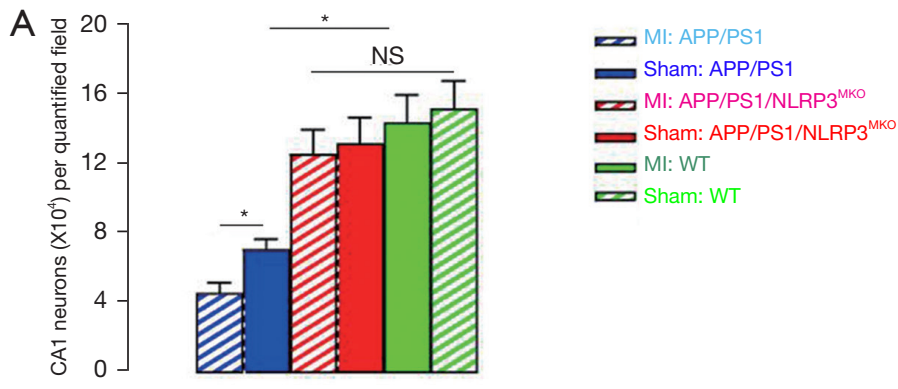

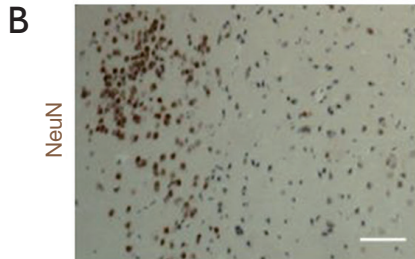

MI: APP/PS1

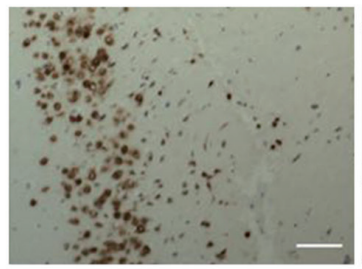

Sham: APP/PS1

C

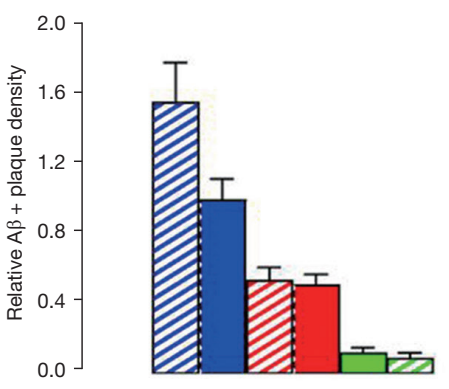

D

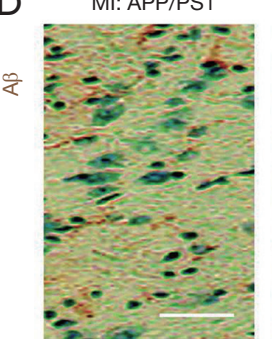

Sham: APP/PS1

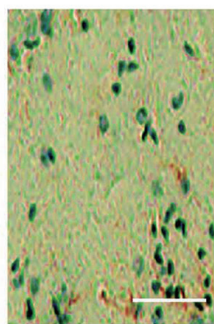

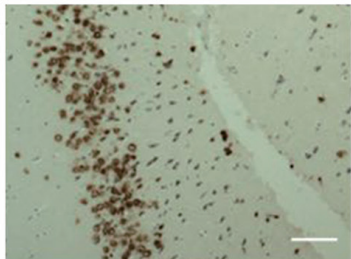

MI: APP/PS1/NLRP3 ${ }^{\text {MKO }}$

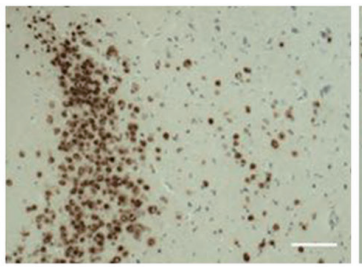

Sham: APP/PS1/NLRP3 ${ }^{\text {MKO }}$

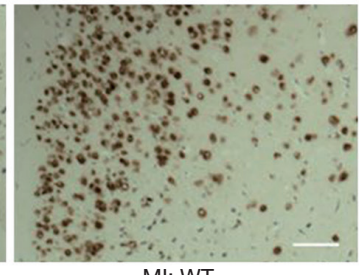

MI: WT

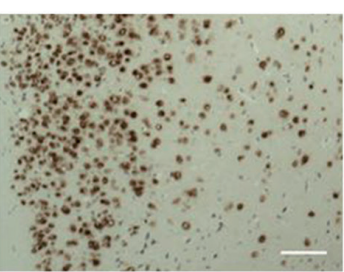

Sham: WT

$$
\begin{aligned}
& \text { MI: APP/PS1 } \\
& \text { Sham: APP/PS1 } \\
& \text { MI: APP/PS1/NLRP3 }{ }^{\text {Mko }} \\
& \text { MI: WT } \\
& \text { Sham: WT }
\end{aligned}
$$

Figure 5 MI-mediated A $\beta$ deposition is NLRP3-dependent. (A,B) We quantified the neuronal loss in the CA1 region of the hippocampus of WT, APP/PS1/NLRP3 ${ }^{M K O}$, and APP/PS1 mice at sacrifice by NeuN staining, shown by quantification (A) and by representative images (B). We found that MI did not significantly alter the neuron density in WT mice. However, MI significantly reduced the neurons in APP/PS1 mice. The neurons in APP/PS1/NLRP $3^{\mathrm{MKO}}$ mice were significantly higher compared to APP/PS1 mice and did not decrease after MI. (C,D) We analyzed the A $\beta$ deposition in the CA1 region of the hippocampus in these mice at sacrifice, shown by quantification of DAB (3,3'-diaminobenzidine) staining for $\mathrm{A} \beta(\mathrm{C})$ and by representative images (D). We found that $\mathrm{MI}$ did not increase $\mathrm{A} \beta$ deposition in WT mice but significantly increased the A $\beta$ levels in APP/PS1 mice. The A $\beta$ levels in APP/PS1/NLRP3 ${ }^{\mathrm{MKO}}$ mice were significantly lower than those in APP/PS1 mice and did not decrease after MI. *, $\mathrm{P}<0.05$. NS, non-significant. For groups 1 and 2 (WT), N=5. For groups 3-6 (APP/PS1/NLRP3 ${ }^{\mathrm{MKO}}$, APP/PS1), N=9. Scale bars are 50 $\mu \mathrm{m}$. 
of $\mathrm{AD}$; however, "AD mice" cannot replicate the disease completely, which is the main limitation of this study.

However, the current study does provide important information about the role of the NLRP3 inflammasome in the development of $\mathrm{AD}$ in mice, and thus, further investigation into the role of the NLRP3 inflammasome in $\mathrm{AD}$ patients is warranted. For example, studying the correlation between the levels of NLRP 3 activation in brain specimens from dead AD patients and their AD severity levels measured before death could be an attractive approach. Moreover, exploring of incidence of AD development in people with or without a NLRP3 mutation is another interesting topic that could benefit society and/or the scientific community.

\section{Acknowledgments}

Funding: This study was supported by the Natural Science Foundation of Xinjiang Uygur Autonomous Region (No. 2021D01C024).

\section{Footnote}

Reporting Checklist: The authors have completed the ARRIVE reporting checklist. Available at https://dx.doi. org/10.21037/atm-21-4931

Data Sharing Statement: Available at https://dx.doi. org/10.21037/atm-21-4931

Conflicts of Interest: All authors have completed the ICMJE uniform disclosure form (available at https://dx.doi. org/10.21037/atm-21-4931). The authors report funding support from the Natural Science Foundation of Xinjiang Uygur Autonomous Region (No. 2021D01C024). The authors have no other conflicts of interest to declare.

Ethical Statement: The authors are accountable for all aspects of the work in ensuring that questions related to the accuracy or integrity of any part of the work are appropriately investigated and resolved. Experiments were performed under a project license (approved at 03/27/2021, same title with this article) granted by institutional ethics board of Fudan University. All experiments were performed in strict accordance with Guidelines for Laboratory Animal Management issued by Fudan University.

Open Access Statement: This is an Open Access article distributed in accordance with the Creative Commons Attribution-NonCommercial-NoDerivs 4.0 International License (CC BY-NC-ND 4.0), which permits the noncommercial replication and distribution of the article with the strict proviso that no changes or edits are made and the original work is properly cited (including links to both the formal publication through the relevant DOI and the license). See: https://creativecommons.org/licenses/by-nc-nd/4.0/.

\section{References}

1. Toepper M. Dissociating Normal Aging from Alzheimer's Disease: A View from Cognitive Neuroscience. J Alzheimers Dis 2017;57:331-52.

2. Hruska-Plochan M, Li B, Kyburz D, et al. New and emerging roles of small RNAs in neurodegeneration, muscle, cardiovascular and inflammatory diseases. Swiss Med Wkly 2015;145:w14192.

3. Licastro F, Chiappelli M, Caldarera CM, et al. Sharing pathogenetic mechanisms between acute myocardial infarction and Alzheimer's disease as shown by partially overlapping of gene variant profiles. J Alzheimers Dis 2011;23:421-31.

4. Sundboll J, Horvath-Puho E, Adelborg K, et al. Higher Risk of Vascular Dementia in Myocardial Infarction Survivors. Circulation 2018;137:567-77.

5. Mrak RE, Griffin WS. Interleukin-1 and the immunogenetics of Alzheimer disease. J Neuropathol Exp Neurol 2000;59:471-6.

6. Buxbaum JD, Oishi M, Chen HI, et al. Cholinergic agonists and interleukin 1 regulate processing and secretion of the Alzheimer beta/A4 amyloid protein precursor. Proc Natl Acad Sci U S A 1992;89:10075-8.

7. Griffin WS, Stanley LC, Ling C, et al. Brain interleukin 1 and S-100 immunoreactivity are elevated in Down syndrome and Alzheimer disease. Proc Natl Acad Sci U S A 1989;86:7611-5.

8. Shaftel SS, Griffin WS, O'Banion MK. The role of interleukin-1 in neuroinflammation and Alzheimer disease: an evolving perspective. J Neuroinflammation 2008;5:7.

9. Rathinam VA, Vanaja SK, Fitzgerald KA. Regulation of inflammasome signaling. Nat Immunol 2012;13:333-42.

10. McGettrick AF, O'Neill LA. NLRP3 and IL-1beta in macrophages as critical regulators of metabolic diseases. Diabetes Obes Metab 2013;15 Suppl 3:19-25.

11. Saresella M, La Rosa F, Piancone F, et al. The NLRP3 and NLRP1 inflammasomes are activated in Alzheimer's disease. Mol Neurodegener 2016;11:23. 
12. Dempsey C, Rubio Araiz A, Bryson KJ, et al. Inhibiting the NLRP3 inflammasome with MCC950 promotes nonphlogistic clearance of amyloid-beta and cognitive function in APP/PS1 mice. Brain Behav Immun 2017;61:306-16.

13. Jankowsky JL, Fadale DJ, Anderson J, et al. Mutant presenilins specifically elevate the levels of the 42 residue beta-amyloid peptide in vivo: evidence for augmentation of a 42-specific gamma secretase. Hum Mol Genet 2004;13:159-70.

14. Kaiser T, Feng G. Tmem119-EGFP and Tmem119CreERT2 Transgenic Mice for Labeling and Manipulating Microglia. eNeuro 2019;6.

15. Brydges SD, Mueller JL, McGeough MD, et al. Inflammasome-mediated disease animal models reveal roles for innate but not adaptive immunity. Immunity 2009;30:875-87.

16. Mezzaroma E, Toldo S, Abbate A. Role of NLRP3 (cryopyrin) in acute myocardial infarction. Cardiovasc Res 2013;99:225-6.

17. Toldo S, Abbate A. The NLRP3 inflammasome in acute myocardial infarction. Nat Rev Cardiol 2018;15:203-14.

18. Zhang W, Luo P. Myocardial Infarction Predisposes Neurodegenerative Diseases. J Alzheimers Dis 2020;74:579-87.

Cite this article as: Cheng $\mathrm{K}$, Wang J, Chen Q, Zhao G, Pang Y, Xu Y, Ge J, Zhu W. Inflammasome-mediated neurodegeneration following heart disease. Ann Transl Med 2021;9(20):1560. doi: 10.21037/atm-21-4931
19. Scott TM, Bhadelia RA, Qiu WQ, et al. Small Vessel Cerebrovascular Pathology Identified by Magnetic Resonance Imaging Is Prevalent in Alzheimer's Disease and Mild Cognitive Impairment: A Potential Target for Intervention. J Alzheimers Dis 2018;65:293-302.

20. van Middelaar T, van Dalen JW, van Gool WA, et al. Visit-To-Visit Blood Pressure Variability and the Risk of Dementia in Older People. J Alzheimers Dis 2018;62:727-35.

21. Li L, Ismael S, Nasoohi S, et al. Thioredoxin-Interacting Protein (TXNIP) Associated NLRP3 Inflammasome Activation in Human Alzheimer's Disease Brain. J Alzheimers Dis 2019;68:255-65.

22. La Rosa F, Saresella M, Marventano I, et al. Stavudine Reduces NLRP3 Inflammasome Activation and Modulates Amyloid-beta Autophagy. J Alzheimers Dis 2019;72:401-12.

23. Ruan C, Sun L, Kroshilina A, et al. A novel Tmem119tdTomato reporter mouse model for studying microglia in the central nervous system. Brain Behav Immun 2020;83:180-91.

(English Language Editor: A. Kassem) 
A

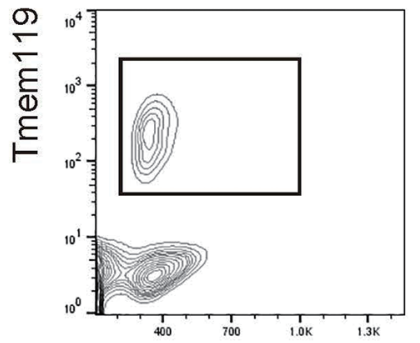

FSC

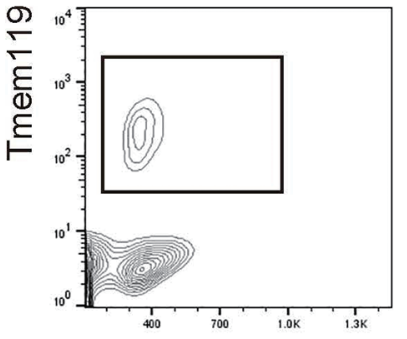

FSC

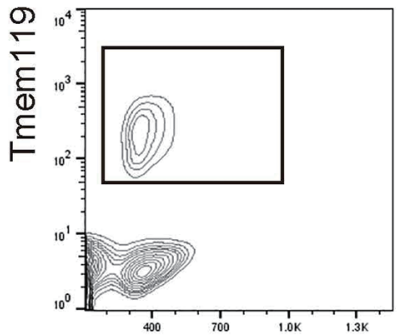

FSC
WT

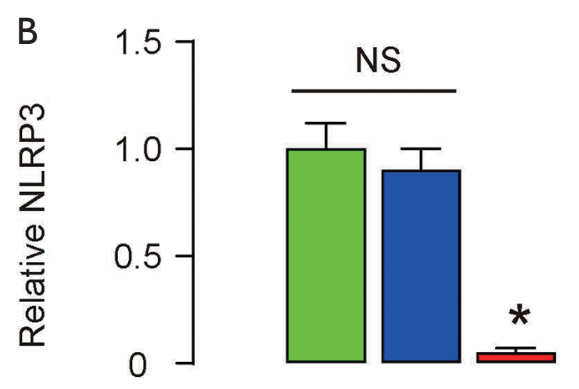

APP/PS1

Figure S1 Validation of NLRP3 knockout in microglia from APP/PS1/NLRP3MKO mice. (A) Microglia in the brain digests from 3 experimental mouse strains prepared by a 30-minutes' treatment with $0.25 \%$ trypsin were isolated based on their expression of Tmem119. (B) ELISA was used to examine for NLRP3 levels, which confirmed the knockdown of NLRP3 specifically in APP/PS1/NLRP3MKO mice. *, $\mathrm{P}<0.05$. NS, non-significant. 